\title{
Nomograms based on clinicopathological factors and inflammatory indicators for prediction of early and late recurrence of hepatocellular carcinoma after surgical resection for patients with chronic hepatitis B
}

\author{
Tianxing Dai ${ }^{1 \#}$, Mingbin Deng ${ }^{1 \#}$, Linsen $\mathrm{Ye}^{1 \#}$, Guozhen $\mathrm{Lin}^{1}$, Rongqiang Liu ${ }^{1}$, Yinan Deng ${ }^{1,2}, \mathrm{Rong}^{13}$, \\ Wei Liu ${ }^{3}$, Hua Li ${ }^{1,2}$, Yang Yang ${ }^{1,2}$, Guihua Chen ${ }^{1,2}$, Guoying Wang ${ }^{1,2}$ \\ ${ }^{1}$ Department of Hepatic Surgery and Liver Transplant Program, The Third Affiliated Hospital of Sun Yat-Sen University, Guangzhou, China; \\ ${ }^{2}$ Organ Transplantation Institute of Sun Yat-Sen University, Guangzhou, China; ${ }^{3}$ Guangdong Key Laboratory of Liver Disease Research, The Third \\ Affiliated Hospital of Sun Yat-Sen University, Guangzhou, China \\ Contributions: (I) Conception and design: T Dai, G Wang; (II) Administrative support: Y Yang, G Chen, G Wang; (III) Provision of study materials or \\ patients: H Li, W Liu, Y Yang, G Chen, G Wang; (IV) Collection and assembly of data: T Dai, M Deng, R Liu; (V) Data analysis and interpretation: \\ T Dai, L Ye, Y Deng, R Li; (VI) Manuscript writing: All authors; (VII) Final approval of manuscript: All authors. \\ \#These authors contributed equally to this work. \\ Correspondence to: Guoying Wang. Department of Hepatic Surgery and Liver Transplant Program, The Third Affiliated Hospital of Sun Yat-Sen \\ University, 600 Tianhe Road, Guangzhou 510630, China. Email: wanggy3@126.com.
}

Background: Few studies have focused on the prognostic values of inflammation-related factors for different phases of recurrence in hepatocellular carcinoma (HCC). We aimed to identify the different risk factors for overall, early, and late recurrence, and to establish nomograms based on inflammation-related parameters for predicting the risks of recurrence in a group of HCC patients undergoing hepatectomy.

Methods: We retrospectively enrolled 383 HCC patients with chronic hepatitis B (CHB) who underwent hepatectomy. Univariate and multivariate Cox analyses were conducted to identify independent risk factors for recurrence. Nomograms for overall, early, and late recurrence-free survival (RFS) were established. The discrimination and calibration abilities of the nomograms were evaluated by concordance indexes (C-index), calibration plots, and Kaplan-Meier curves. Finally, receiver operating characteristic (ROC) curves were used to compare the derived nomograms with other existing models.

Results: Fibrinogen, lymphocyte-to-monocyte ratio, and S-index inflammation-related factors were independently related to overall and early RFS, but only the S-index correlated with late recurrence. Nomograms with tumor number, diameter, and pathological differentiation for overall and early RFS were established, while nomogram for late recurrence was constructed with tumor number and Child-Pugh grade. The C-indexes for overall, early, and late RFS were $0.679,0.677$, and 0.728 , respectively. The calibration plots fit well. The nomograms showed superior discrimination capacities and better performance prediction with larger areas under the curve for recurrence.

Conclusions: The developed nomograms that integrated inflammation-related factors showed high predictive accuracy for overall, early, and late recurrence in HCC patients with CHB after hepatectomy.

Keywords: Hepatocellular carcinoma (HCC); inflammation; nomogram; prognosis; recurrence

Submitted Feb 05, 2020. Accepted for publication Sep 29, 2020.

doi: 10.21037/atm-20-1353

View this article at: http://dx.doi.org/10.21037/atm-20-1353

(c) Annals of Translational Medicine. All rights reserved. 


\section{Introduction}

Hepatocellular carcinoma (HCC) is one of the leading causes of cancer death worldwide and accounted for almost 746,000 deaths in 2012 (1). In China, the morbidity and mortality rates of HCC remain at high levels with nearly half of the global new cases and deaths, mostly because of the prevalence of chronic hepatitis $\mathrm{B}(\mathrm{CHB})$ and progressive cirrhosis $(1,2)$. Despite the remarkable advances in anticancer treatments, surgical resection remains the firstline treatment and is regarded as a curative therapy for patients with resectable HCC $(1,3)$. Surgical resection is widely performed in China, and not limited to patients with Barcelona Clinic Liver Cancer (BCLC) 0-A stage. However, the long-term outcomes of these patients remain unsatisfying mainly because of the high incidence of recurrence (4). With the different mechanisms of recurrence being taken into account, the classification of early and late recurrence of HCC has been proposed $(5,6)$. Theoretically, occult metastasis is mainly responsible for early recurrence, while de novo HCC arising from background liver diseases mainly contributes to late recurrence. Hence, different risk factors are presumed to relate to each type of recurrence. Previous studies have revealed that the tumor's pathological characteristics, including size, number, stage, vascular invasion, surgical margin, and pathological differentiation are significantly related to early HCC recurrence, whereas cirrhosis has notably been shown to be associated with late recurrence (5-7). Thus, HCC patients with different recurrence will have differing prognoses, and identifying and utilizing clinically available risk factors would be of considerable value in accurately assessing the risk of recurrence in HCC patients. Accordingly, an individualized follow-up schedule and prevention program will further improve the prognosis of these patients.

There is growing evidence for the significant role of inflammation in tumor development, progression, and metastasis (8). Various hematological and biochemical parameters have been identified, such as neutrophils, lymphocytes, platelets, monocytes, fibrinogen, and c-reactive protein (CRP), which are considered adequate for detecting and evaluating the status of an inflammatory response (9). Other studies have also demonstrated the prognostic roles of these parameters in various tumors (10). However, given the complexity and heterogeneity of tumors, the use of individual indicators is self-limiting in assessing the overall picture of inflammation. On that note, researchers have proposed a series of composite indexes, including the neutrophil-to- lymphocyte ratio (NLR), derived NLR (dNLR), plateletto-lymphocyte ratio (PLR), lymphocyte-to-monocyte ratio (LMR), and systemic immune-inflammation index (SII), which have shown better performance in evaluating inflammation and predicting prognosis (11-14). The existence of background liver diseases (especially viral hepatitis or cirrhosis) means HCC is usually considered to be an inflammation-related malignancy and represents a special inflammatory microenvironment and response. In light of this, parameters associated with inflammationmediated impairment of liver function, such as the aspartate transaminase-to-platelet count ratio index (APRI), aspartate transaminase-to-neutrophil ratio index (ANRI), gammaglutamyl transpeptidase-to-platelet ratio (GPR), fibrosis index based on four factors (FIB-4), and the S-index, might provide an appropriate overview of the specific locoregional inflammatory response in the liver, and their prognostic values for patients with HCC have also been evaluated in different studies (15-17). However, few studies focused on the integrated analysis of both the systemic and the locoregional inflammatory response in predicting patients' outcomes after treatments.

Hence, the present study aimed to identify the different risk factors for overall, early, and late recurrence, and to thereby establish nomograms based on inflammation-related parameters for predicting the risk of HCC recurrence after surgical resection in a group of patients with CHB. Finally, the prognostic prediction efficiency was evaluated, and patients were divided into different risk groups accordingly. We present the following article in accordance with the STROBE reporting checklist (available at http://dx.doi. org/10.21037/atm-20-1353).

\section{Methods}

\section{Patients cohort}

From March 2005 to August 2017, patients who were diagnosed with HCC in our department at The Third Affiliated Hospital of Sun Yat-Sen University were retrospectively reviewed. These patients were further screened with the following inclusion criteria to establish the study cohort: (I) pathological confirmation of HCC; (II) without extrahepatic or distant metastases; (III) without prior anti-cancer treatments before surgery; (IV) without other concurrent malignancies; (V) age $\geq 18$ years; (VI) valid and available clinicopathological and follow-up data. Finally, a cohort of 383 HCC patients was enrolled into our study. The study was conducted in accordance with the 
Declaration of Helsinki (as revised in 2013). The study was approved by the institutional ethics board of The Third Affiliated Hospital of Sun Yat-Sen University (No. 202002-119). Informed consent was taken from all the patients.

\section{Clinicopathological data collection}

The medical records were reviewed retrospectively and the clinicopathological data were collected for all patients. For demographics, the gender and age were recorded. The tumor-related information was referred with tumor number, diameter of the largest nodule, and status of vascular invasion, which was reported by preoperative radiological examinations including contrast-enhanced computed tomographic (CT) scans and/or magnetic resonance imaging (MRI). The pathological differentiation was classified as well-, moderately-, and poorly-differentiated types. The etiology of viral hepatitis was also recorded. Patients in the study were all positive for hepatitis B surface antigen. The existence of cirrhosis was mainly determined by histopathological diagnosis. In addition, the Child-Pugh grade and the Model for End-stage Liver Disease (MELD) score were applied to evaluate liver function. The BCLC staging system was adopted to stratify the HCC patients into different tumor stages.

Hematological indexes were obtained from blood test results within one week prior to surgery, and included white blood cell count, neutrophil count, lymphocyte count, monocyte count, platelet count, alanine transaminase (ALT), aspartate transaminase (AST), albumin (ALB), total bilirubin (TBIL), gamma-glutamyl transpeptidase (GGT), creatinine (Cr), fibrinogen, international normalized ratio (INR), $\alpha$-fetoprotein (AFP), and HBV-DNA. Subsequently, the inflammation-related indexes were calculated according to previously published literature, and included NLR (11), dNLR (17), PLR (13), LMR (16), GPR (13), SII (12), FIB-4 (15), S-index (13), prognostic nutritional index (PNI) (13), aspartate transaminase-to-lymphocyte ratio index (ALRI) (13), and albumin-bilirubin (ALBI) (17). The formulas for the models mentioned above are summarized in Table S1.

\section{Follow-up}

After surgery, patients were encouraged to revisit the outpatient clinic for regular checkups (follow-up surveillance and treatments). The follow-up schedule was as follows: serum AFP and liver function were measured monthly in the first year and every 3 months thereafter. Furthermore, abdominal ultrasound and enhanced CT/MRI were performed every 3 months during the first 2 years, and every 6 months thereafter. If recurrence was suspected, chest CT, bone scanning, or positron emission tomography (PET) would be further performed to check recurrence status, and patients were hospitalized for evaluation of treatment, which could include repeat-resection, ablation, transcatheter arterial chemoembolization, radiotherapy, targeted therapy, or supportive management. In the present study, all cases of recurrence were confirmed by existence of intrahepatic lesions on CT/MRI, regardless of the extrahepatic failure. The recurrence-free survival (RFS) was defined as the interval between the date of surgery and the date of a confirmed recurrence. If recurrence had not occurred within the follow-up time of this study, the cases were classified as censored on the date of death or the last date of follow-up.

\section{Statistical analysis}

Quantitative variables are described as median and interquartile range, and categorical variables are presented as count and percentages. The cut-off values of the calculated inflammation-related parameters (NLR, dNLR, PLR, LMR, GPR, SII, PNI, APRI, ANRI, ALRI, ALBI, FIB-4, and S-index), fibrinogen, and MELD score were determined by receiver operating characteristic (ROC) curve with the best Youden's index, while the cut-off values of tumor diameter, AFP, and HBV-DNA were determined by routine clinical practice.

The RFS was calculated by the Kaplan-Meier method and compared by log-rank test between different groups. Univariate Cox analysis was conducted to evaluate the potentially related factors for tumor recurrence, and then variables with $\mathrm{P}$ values $<0.1$ were subjected to multivariate Cox proportional hazards analysis for further assessment of their independent prognostic effects. The stepwise forward selection method was adopted, and the hazard ratio (HR) and $95 \%$ confidence interval (CI) were calculated. Notably, we first investigated those factors contributing to general recurrence with overall RFS, followed by those contributing to early and late recurrence respectively, with the cut-off time point of 2 years after surgery. As for early recurrence, the status of patients was determined at 24 months after surgery, and patients without recurrence were marked as censored for analysis. For the analysis of late recurrence, only patients with a follow-up time over 24 months and no 
Table 1 Baseline characteristics of HCC patients

\begin{tabular}{|c|c|}
\hline Category & Median (range)/N (\%) \\
\hline Gender (male/female) & $337(88.0 \%) / 46(12.0 \%)$ \\
\hline Age, years & $50(41,59)$ \\
\hline Smoke (yes/no) & $128(33.4 \%) / 255$ (66.6\%) \\
\hline Drink (yes/no) & $76(19.8 \%) / 307$ (80.2\%) \\
\hline Diabetes (yes/no) & $34(8.9 \%) / 349(91.1 \%)$ \\
\hline Cirrhosis (yes/no) & $253(66.1 \%) / 130$ (33.9\%) \\
\hline Ascites (yes/no) & $45(11.7 \%) / 338(88.3 \%)$ \\
\hline Tumor diameter, $\mathrm{cm}$ & $4.3(3.0,7.3)$ \\
\hline Tumor number (multiple/single) & 113 (29.5\%)/270 (70.5\%) \\
\hline Vascular invasion (yes/no) & 138 (36.0\%)/245 (64.0\%) \\
\hline Child-Pugh grade $(B / A)$ & $25(6.5 \%) / 358(93.5 \%)$ \\
\hline MELD score & $5(3,7)$ \\
\hline BCLC stage $(B-C / 0 \sim A)$ & $209(54.6 \%) / 174(45.4 \%)$ \\
\hline $\begin{array}{l}\text { Pathological differentiation } \\
\text { (poor-moderate/well) }\end{array}$ & $315(82.2 \%) / 68(17.8 \%)$ \\
\hline AFP, ng/mL (>400/s400) & $123(32.1 \%) / 260$ (67.9\%) \\
\hline $\begin{array}{l}\text { HBV-DNA, copies/mL } \\
(>1,000 / \leq 1,000)\end{array}$ & $181(47.3 \%) / 202(52.7 \%)$ \\
\hline Fibrinogen, $\mathrm{g} / \mathrm{mL}$ & $3.02(2.49,3.65)$ \\
\hline NLR & $1.90(1.45,2.70)$ \\
\hline dNLR & $1.49(1.08,2.15)$ \\
\hline PLR & $99.49(71.66,135.20)$ \\
\hline LMR & $3.92(2.83,5.04)$ \\
\hline GPR & $0.35(0.21,0.71)$ \\
\hline SII & $316.80(187.83,490.00)$ \\
\hline PNI & $48,90(45.35,52.40)$ \\
\hline APRI & $0.23(0.15,0.35)$ \\
\hline ANRI & $11.91(8.26,17.89)$ \\
\hline ALRI & $23.50(17.88,31.68)$ \\
\hline ALBI & $-2.68(-2.90,-2.43)$ \\
\hline FIB-4 & $1.76(1.19,3.12)$ \\
\hline S-index & $0.21(0.13,0.46)$ \\
\hline Recurrence (no/early/late) & $\begin{array}{l}154(40.2 \%) / 198(51.7 \%) / 31 \\
(8.1 \%)\end{array}$ \\
\hline
\end{tabular}

MELD, Model for End-stage Liver Disease; BCLC, Barcelona Clinic Liver Cancer; AFP, $\alpha$-fetoprotein; HBV, hepatitis B virus; NLR, neutrophil-to-lymphocyte ratio; dNLR, derived neutrophilto-lymphocyte ratio; PLR, platelet-to-lymphocyte ratio; LMR, lymphocyte-to-monocyte ratio; GPR, gamma-glutamyl transpeptidase-to-platelet ratio; SII, systemic immune-inflammation index; PNI, prognostic nutritional index; APRI, aspartate transaminase-to-platelet count ratio index; ANRI, aspartate transaminase-to-neutrophil ratio index; ALRI, aspartate transaminase-to-lymphocyte ratio index; ALBI, albumin-bilirubin; FIB-4, fibrosis index based on the four factors. early recurrence were included. The survival curves were plotted using the "survival" and "survminer" packages in R software.

To estimate the overall, early, and late RFS, nomograms based on the results of multivariate analysis were constructed using the "rms" package in R software. Prediction performance was evaluated by the concordance index (C-index) and a calibration curve with the derivation and validation sets based on a 1000-resampled bootstrap. According to the formulized nomograms, each patient would have a total score (NomoScore) for risk stratification of recurrence. Patients were also divided into different risk groups with the cut-off points automatically determined by the X-tile software (version 3.6.1; Yale University, New Haven, CT, USA). Survival analysis was also conducted to compare recurrence in the different risk groups with the above-mentioned methods. Finally, we compared the prediction performance of our nomograms with other prognostic variables and models by ROC curves with the area under the curves (AUCs).

All statistical analyses were conducted by SPSS software (version 22.0; BM Corp., Armonk, NY, USA) and R project (version 3.4.2; R Foundation for Statistical Computing, Vienna, Austria). A P value $<0.05$ (two-tailed) was considered statistically significant.

\section{Results}

\section{Baseline characteristics of enrolled patients with HCC}

A total of 383 patients with HCC were enrolled, and the baseline characteristics are summarized in Table 1. The median age of all patients was 50 years (range, $41-59$ years) and $337(88.0 \%)$ were male. Eight patients had coinfection with $\mathrm{HCV}$, and only one patient was coinfected with HIV. Based on this, hepatitis induced cirrhosis was confirmed pathologically in $253(66.1 \%)$ patients, while most patients $(358,93.5 \%)$ showed well-preserved liver function with child-Pugh grade A. Hence, the MELD score, another model for evaluating liver function, also showed a low level with a median of 5 (range, 3-7). As for the tumor features, the median tumor diameter was $4.3 \mathrm{~cm}(3.0-7.3 \mathrm{~cm}), 70.5 \%$ of patients had only one nodule, and vascular invasion was found in 138 cases $(36.0 \%)$. According to the BCLC staging system, 174 (45.4\%) patients were in the very early and early stages (0-A), and $209(54.6 \%)$ patients were in the intermediate and advanced stage (B: 71, 18.5\%; C: 138 , $36.1 \%)$. All patients underwent surgical resection as their 

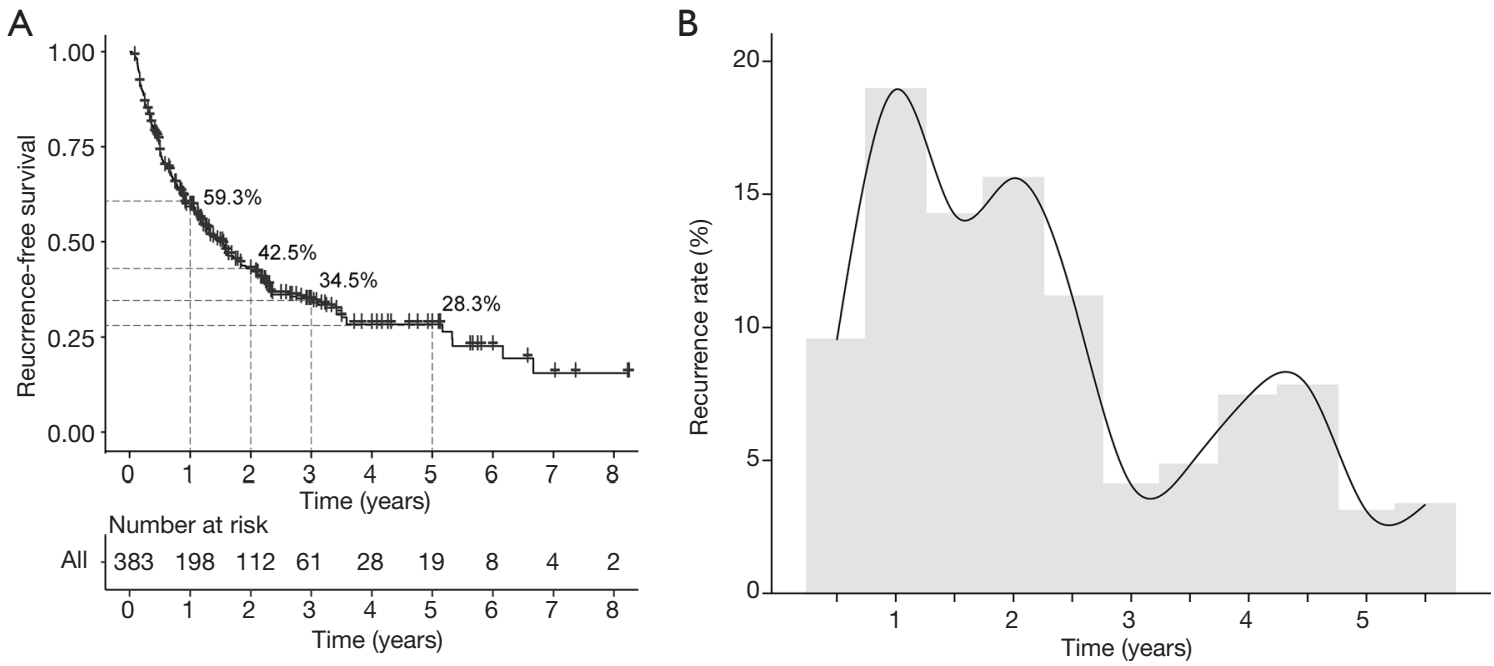

Figure 1 Overall cumulative RFS (A) and the recurrence rate curve (B) for the whole cohort of HCC patients after hepatectomy. RFS, recurrence-free survival.

first treatments for HCC, and recurrences occurred in 229 (59.8\%) patients (early recurrence, 198 ; late recurrence, 31 ) during the follow-up period.

\section{Prognostic factors for overall RFS of HCC patients and nomogram construction}

The cumulative 1-, 2-, 3-, and 5-year overall RFS rates for all HCC patients were $59.3 \%, 42.5 \%, 34.5 \%$, and $28.3 \%$, respectively (Figure 1A). The recurrence rate curve (Figure 1B) depicts the dynamic changes in the recurrence rate during the follow-up period, showing two high peaks in the first 2 years, followed by another relatively low peak within 4-5 years after surgery, representing early and late recurrence respectively in the HCC patients who underwent resection.

First, we evaluated the prognostic factors related to overall RFS. The results of univariate analysis showed that smoking $(\mathrm{P}=0.014)$, diabetes $(\mathrm{P}=0.049)$, tumor diameter $(\mathrm{P}<0.001)$, tumor number $(\mathrm{P}<0.001)$, vascular invasion $(\mathrm{P}<0.001)$, BCLC stage $(\mathrm{P}<0.001)$, pathological differentiation $(\mathrm{P}<0.001)$, AFP $(\mathrm{P}=0.007)$, fibrinogen $(\mathrm{P}<0.001)$, LMR $(\mathrm{P}<0.001)$, GPR $(\mathrm{P}<0.001)$, SII $(\mathrm{P}=0.021)$, PNI $(\mathrm{P}=0.001)$, APRI $(\mathrm{P}=0.003)$, ANRI (0.018), ALBI $(\mathrm{P}<0.001)$, FIB-4 $(0.036)$, and $\mathrm{S}$-index $(\mathrm{P}<0.001)$ were significantly related to the overall RFS. Next, the above factors (all $\mathrm{P}<0.05)$, combined with NLR $(\mathrm{P}<0.1)$ and ALRI $(\mathrm{P}<0.1)$, were entered into the multivariate analysis, and the results showed that tumor diameter $(\mathrm{P}=0.008$;
HR: 1.471 , $95 \%$ CI: 1.106-1.958), tumor number $(\mathrm{P}<0.001$; HR: 2.113, 95\% CI: 1.610-2.772), pathological differentiation ( $\mathrm{P}=0.010$; HR: 1.688, 95\% CI: 1.136-2.507), fibrinogen ( $\mathrm{P}=0.007$; HR: $1.519,95 \% \mathrm{CI}: 1.121-2.058)$, LMR ( $\mathrm{P}=0.046$; HR: 1.324 , 95\% CI: $1.005-1.745)$, and S-index ( $\mathrm{P}<0.001$; HR: 1.813, 95\% CI: $1.385-2.374)$ were independent factors for overall RFS (Table 2).

Based on these six significant prognostic factors, a nomogram was developed to predict the 1-, 3-, and 5-year overall RFS rates for HCC patients (Figure $2 A$ ). The model exhibited good efficacy in estimating postoperative recurrence with a high $\mathrm{C}$-index of 0.679 . The calibration curves for the 1-, 3- and 5-year RFS rates were largely overlapped with its standard lines (Figure 2B,C,D).

\section{Prognostic factors for early RFS of HCC patients and nomogram construction}

Early recurrence was observed in 198 patients within 2 years after surgery. Univariate analysis identified 17 variables as factors related to early recurrence, including smoking $(\mathrm{P}=0.026)$, diabetes $(\mathrm{P}=0.014)$, tumor diameter $(\mathrm{P}<0.001)$, tumor number $(\mathrm{P}<0.001)$, vascular invasion $(\mathrm{P}<0.001)$, BCLC stage $(\mathrm{P}<0.001)$, pathological differentiation $(\mathrm{P}<0.001)$, AFP $(\mathrm{P}=0.001)$, fibrinogen $(\mathrm{P}<0.001)$, LMR $(\mathrm{P}<0.001)$, GPR $(\mathrm{P}=0.003)$, SII $(\mathrm{P}=0.013)$, $\mathrm{PNI}(\mathrm{P}=0.006)$, APRI $(\mathrm{P}=0.011)$, ANRI $(\mathrm{P}=0.028)$, ALBI $(\mathrm{P}=0.005)$, and $\mathrm{S}$-index $(\mathrm{P}<0.001)($ Table 3). Further multivariate analysis enrolled both above mentioned significant factors and 
Table 2 Univariate and multivariate COX analyses for overall RFS of HCC patients

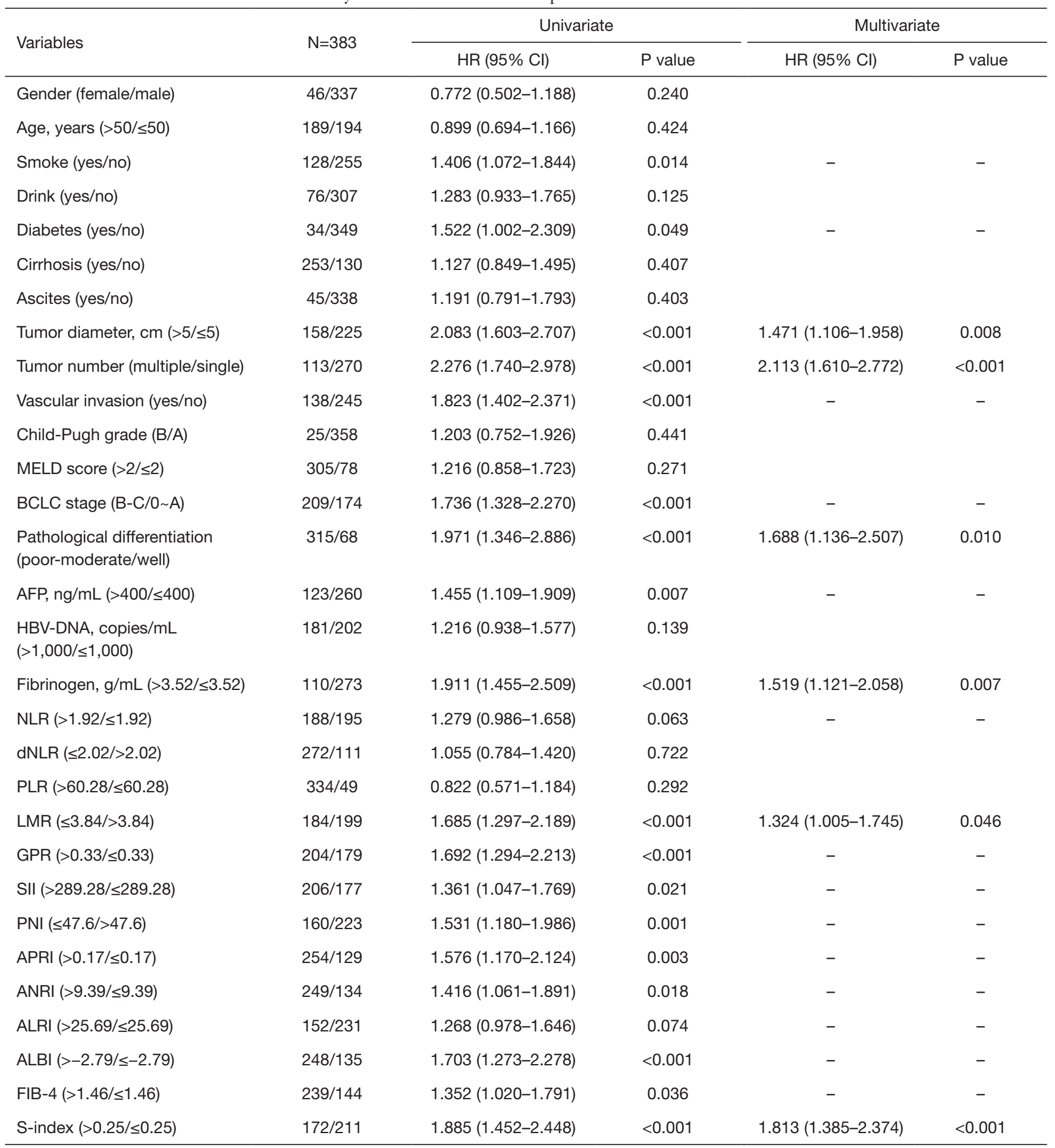

MELD, Model for End-stage Liver Disease; BCLC, Barcelona Clinic Liver Cancer; AFP, $\alpha$-fetoprotein; HBV, hepatitis B virus; NLR, neutrophil-to-lymphocyte ratio; dNLR, derived neutrophil-to-lymphocyte ratio; PLR, platelet-to-lymphocyte ratio; LMR, lymphocyteto-monocyte ratio; GPR, gamma-glutamyl transpeptidase-to-platelet ratio; SII, systemic immune-inflammation index; PNI, prognostic nutritional index; APRI, aspartate transaminase-to-platelet count ratio index; ANRI, aspartate transaminase-to-neutrophil ratio index; ALRI, aspartate transaminase-to-lymphocyte ratio index; ALBI, albumin-bilirubin; FIB-4, fibrosis index based on the four factors; RFS, recurrence-free survival. 
A

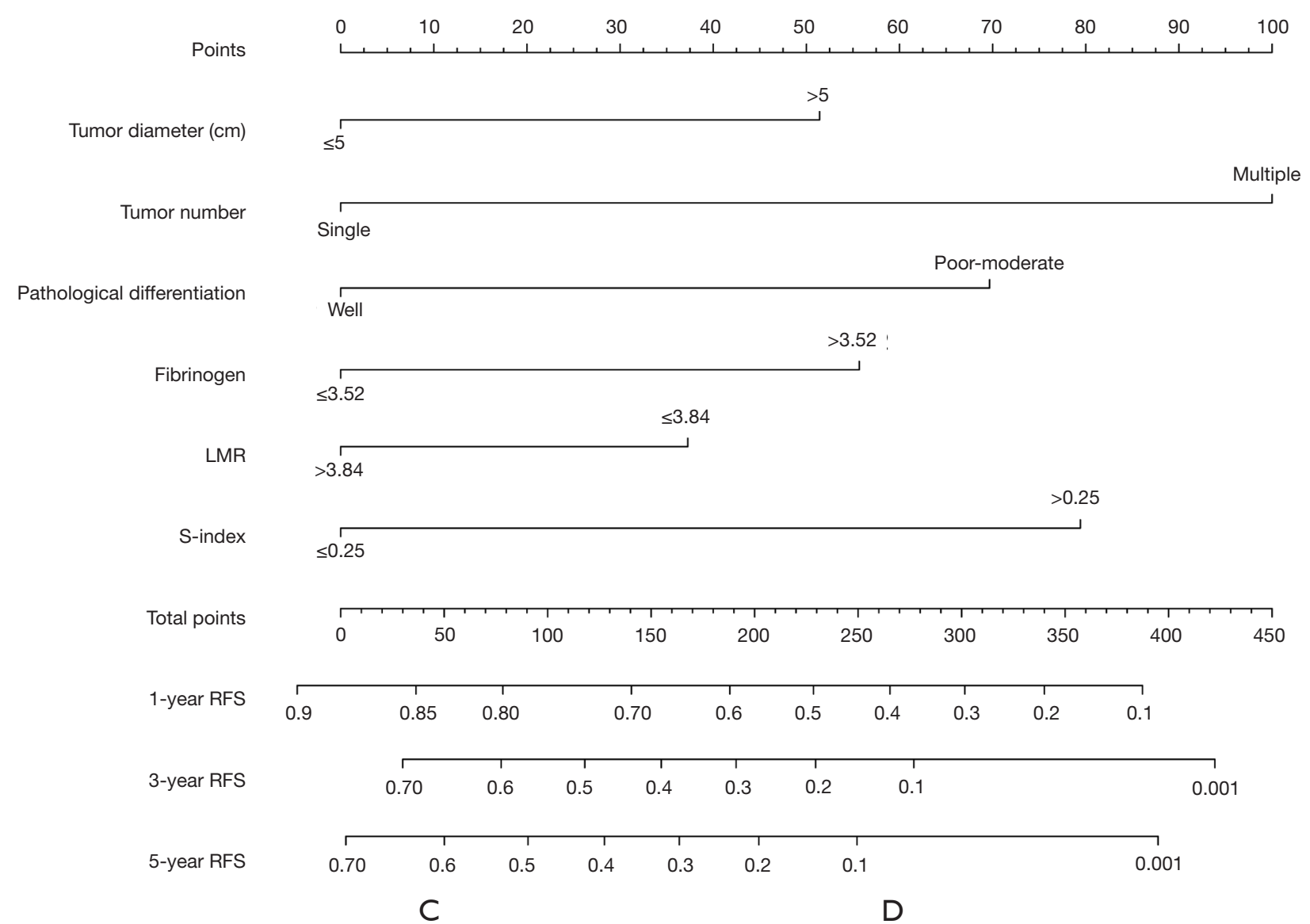

B

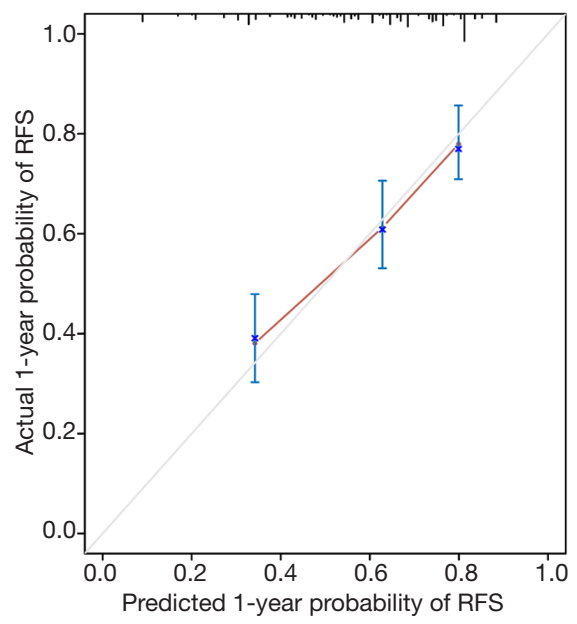

C

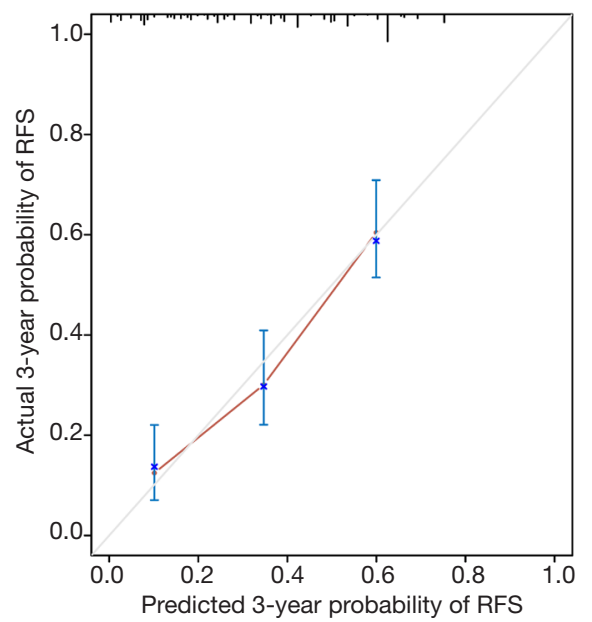

D

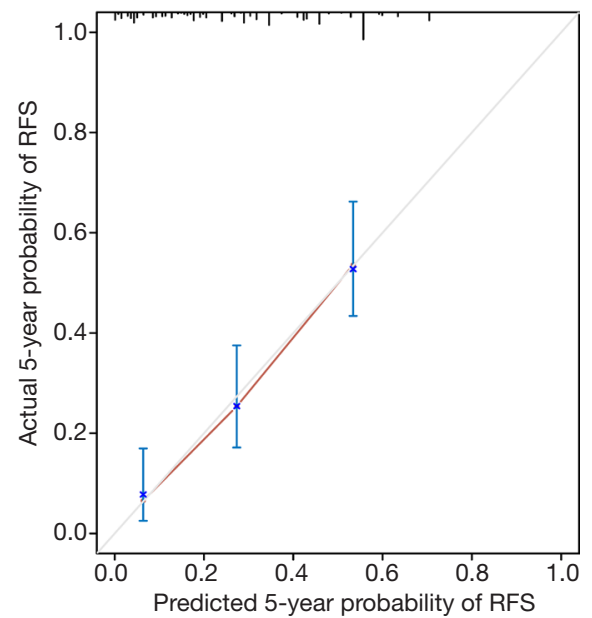

Figure 2 Nomogram for predicting the overall RFS (A) and the calibration curves for predicting the 1-, 3-, and 5-year overall RFS (B, C, and D) in HCC patients who received hepatectomy. RFS, recurrence-free survival; HCC, hepatocellular carcinoma. 
Table 3 Univariate and multivariate COX analyses for early RFS of HCC patients

\begin{tabular}{|c|c|c|c|c|c|}
\hline Variables & $\mathrm{N}=383$ & \multicolumn{2}{|c|}{ Univariate } & \multicolumn{2}{|c|}{ Multivariate } \\
\hline Gender (female/male) & $46 / 337$ & $0.823(0.524-1.295)$ & 0.4 & & \\
\hline Age, years $(>50 / \leq 50)$ & $189 / 194$ & $0.849(0.642-1.122)$ & 0.25 & & \\
\hline Smoke (yes/no) & $128 / 255$ & $1.390(1.041-1.855)$ & 0.026 & - & - \\
\hline Diabetes (yes/no) & $34 / 349$ & $1.706(1.112-2.617)$ & 0.014 & - & - \\
\hline Cirrhosis (yes/no) & $253 / 130$ & $1.104(0.815-1.495)$ & 0.524 & & \\
\hline Ascites (yes/no) & $45 / 338$ & $1.254(0.824-1.907)$ & 0.291 & & \\
\hline Tumor diameter, $\mathrm{cm}(>5 / \leq 5)$ & $158 / 225$ & $2.121(1.603-2.807)$ & $<0.001$ & $1.526(1.125-2.071)$ & 0.007 \\
\hline Child-Pugh grade $(B / A)$ & $25 / 358$ & $0.969(0.563-1.669)$ & 0.910 & & \\
\hline MELD score $(>2 / \leq 2)$ & $305 / 78$ & $1.215(0.836-1.766)$ & 0.307 & & \\
\hline BCLC stage $(B-C / 0 \sim A)$ & $209 / 174$ & $1.694(1.269-2.259)$ & $<0.001$ & - & - \\
\hline $\begin{array}{l}\text { Pathological differentiation (poor- } \\
\text { moderate/well) }\end{array}$ & $315 / 68$ & $2.387(1.503-3.791)$ & $<0.001$ & $1.924(1.199-3.089)$ & 0.007 \\
\hline AFP, ng/mL $(>400 / \leq 400)$ & $123 / 260$ & $1.635(1.227-2.178)$ & 0.001 & - & - \\
\hline HBV-DNA, copies/mL $(>1,000 / \leq 1,000)$ & $181 / 202$ & $1.258(0.952-1.662)$ & 0.106 & & \\
\hline Fibrinogen, g/mL (>3.52/ $\leq 3.52)$ & $110 / 273$ & $1.882(1.408-2.517)$ & $<0.001$ & $1.449(1.048-2.002)$ & 0.025 \\
\hline SII (>289.28/ $\leq 289.28)$ & $206 / 177$ & $1.430(1.078-1.898)$ & 0.013 & - & - \\
\hline $\mathrm{PNI}(\leq 47.6 />47.6)$ & $160 / 223$ & $1.477(1.117-1.953)$ & 0.006 & - & - \\
\hline APRI $(>0.17 / \leq 0.17)$ & $254 / 129$ & $1.510(1.100-2.072)$ & 0.011 & - & - \\
\hline ANRI $(>9.39 / \leq 9.39)$ & $249 / 134$ & $1.417(1.038-1.934)$ & 0.028 & - & - \\
\hline ALRI (>25.69/ $\leq 25.69)$ & $152 / 231$ & $1.285(0.972-1.700)$ & 0.079 & - & - \\
\hline ALBI $(>-2.79 / \leq-2.79)$ & $248 / 135$ & $1.555(1.141-2.121)$ & 0.005 & - & - \\
\hline FIB-4 (>1.46/ $\leq 1.46)$ & $239 / 144$ & $1.252(0.929-1.686)$ & 0.140 & & \\
\hline S-index $(>0.25 / \leq 0.25)$ & $172 / 211$ & $1.744(1.318-2.308)$ & $<0.001$ & 1.655 (1.240-2.208) & 0.001 \\
\hline
\end{tabular}

MELD, Model for End-stage Liver Disease; BCLC, Barcelona Clinic Liver Cancer; AFP, $\alpha$-fetoprotein; HBV, hepatitis B virus; NLR, neutrophil-to-lymphocyte ratio; dNLR, derived neutrophil-to-lymphocyte ratio; PLR, platelet-to-lymphocyte ratio; LMR, lymphocyteto-monocyte ratio; GPR, gamma-glutamyl transpeptidase-to-platelet ratio; SII, systemic immune-inflammation index; PNI, prognostic nutritional index; APRI, aspartate transaminase-to-platelet count ratio index; ANRI, aspartate transaminase-to-neutrophil ratio index; ALRI, aspartate transaminase-to-lymphocyte ratio index; ALBI, albumin-bilirubin; FIB-4, fibrosis index based on the four factors; RFS, recurrence-free survival. 
variables with $\mathrm{P}<0.1$ (NLR, and $\mathrm{ALRI}$ ), and the results showed that tumor diameter $(\mathrm{P}=0.007$; HR: $1.526,95 \% \mathrm{CI}$ : 1.125-2.071), tumor number $(\mathrm{P}<0.001$; HR: $1.910,95 \% \mathrm{CI}$ : 1.432-2.547), pathological differentiation ( $\mathrm{P}=0.007$; HR: 1.924, 95\% CI: 1.199-3.089), fibrinogen ( $\mathrm{P}=0.025$; HR: 1.449, 95\% CI: 1.048-2.002), LMR ( $\mathrm{P}=0.045$; HR: 1.353 , 95\% CI: 1.007-1.817), and S-index ( $\mathrm{P}=0.001$; HR: 1.655 , 95\% CI: 1.240-2.208) were independent prognostic factors for early RFS (Table 3).

A nomogram to evaluate the probability of 1 -, and 2 -year early RFS was constructed on the basis of the above six parameters (Figure $3 A$ ). The nomogram also showed favorable ability in predicting early recurrence with a C-index of 0.677. The calibration curves for 1-, and 2-year early RFS rates were also well-fitted with the standard lines (Figure 3B,C).

\section{Prognostic factors for late RFS of HCC patients and nomogram construction}

Factors related to late recurrence were further examined in the 111 patients who were confirmed to be without recurrence at 2 years after surgery. Recurrence was detected in 31 patients during the follow-up period. Tumor number $(\mathrm{P}<0.001)$, Child-Pugh grade $(\mathrm{P}=0.010), \mathrm{GPR}(\mathrm{P}=0.005)$, ALBI $(\mathrm{P}=0.009)$, and $\mathrm{S}$-index $(\mathrm{P}=0.002)$ were found to be related to late recurrence of $\mathrm{HCC}$ (Table 4). Combined with BCLC stage, fibrinogen, PNI, APRI, and FIB-4 (all $\mathrm{P}<0.1$, these factors were further screened by multivariate analysis, and only tumor number $(\mathrm{P}<0.001$; HR: 4.868, 95\% CI: 2.264-10.467), Child-Pugh grade ( $\mathrm{P}=0.048$; HR: 2.834, 95\% CI: 1.007-7.970), and S-index ( $\mathrm{P}=0.004$; HR: 3.074, 95\% CI: 1.439-6.565) were identified as independent prognostic factors for late recurrence (Table 4).

Another nomogram was built for late recurrence of HCC with the above three variables (Figure $4 A$ ) and the corresponding $\mathrm{C}$-index was 0.728 , which indicated good performance in predicting late recurrence. The calibration curves for the 3-, and 5-year late RFS rates were also wellmatched with the standard lines (Figure 4B,C).

\section{Prognostic performance and risk stratification for recurrence based on the nomogram models}

To further evaluate the discriminatory abilities of the nomograms for overall, early, and late recurrence, patients were divided into different risk groups according to the total scores (NomoScores) calculated by the formulated nomograms (Figure S1). The optimal cut-off points were determined by the X-tile software (Table S2). For overall and early recurrence, patients were classified into three groups: high risk (overall RFS: >280; early RFS: >320), moderate risk (overall RFS: 125-280; early RFS: 222-320), and low risk (overall RFS: $\leq 125$; early RFS: $\leq 222$ ). In consideration of the limited sample size for late recurrence, patients were only divided into two groups: high risk $(>100)$ and low risk $(\leq 100)$. The Kaplan-Meier curves were plotted to compare the RFS rates in the different risk groups, and the results showed a significant distinction ability for recurrence risks in HCC patients based on the NomoScores (Figure 5). Patients with low risks presented with the best overall, early, and late RFS, whereas those with a high risk had the worst overall, early, and late RFS. All P values were $<0.001$.

We also compared the prognostic performance of the nomograms with the BCLC staging system, Child-Pugh grade, MELD score, AFP, and variables involved in creating the nomograms by ROC curve analysis. The AUCs of the nomograms for overall, early, and late RFS were 0.739 (95\% CI: 0.689-0.788, P<0.001), 0.724 (95\% CI: $0.675-$ $0.774, \mathrm{P}<0.001$ ), 0.753 (95\% CI: 0.642-0.863, $\mathrm{P}<0.001$ ), respectively, which were significantly higher than other prognostic models and independent parameters (Figure 6). These results indicated the high discrimination capacity and prognostic accuracy of the nomograms for overall, early and late RFS of HCC patients.

\section{Discussion}

In the present study, we retrospectively investigated the specific factors involved in the overall, early, and late recurrence in HCC patients with $\mathrm{CHB}$ after liver resection. Results showed that the clinicopathological parameters of tumor diameter, number, and pathological differentiation, together with inflammation-related parameters (fibrinogen, LMR, and S-index) were independent factors for the overall RFS. Similarly, six parameters were also found to have a significant association with early recurrence in multivariate analysis, which might be a result of the high incidence of early recurrence in the present cohort (198 cases of early recurrence, and 31 cases of late recurrence). As for late recurrence, tumor number showed a significant prognostic value. In addition, Child-Pugh grade, a widely used parameter for preserved liver function evaluation, which was neither correlated to early nor overall recurrence, was identified as an independent factor for late recurrence. 
A

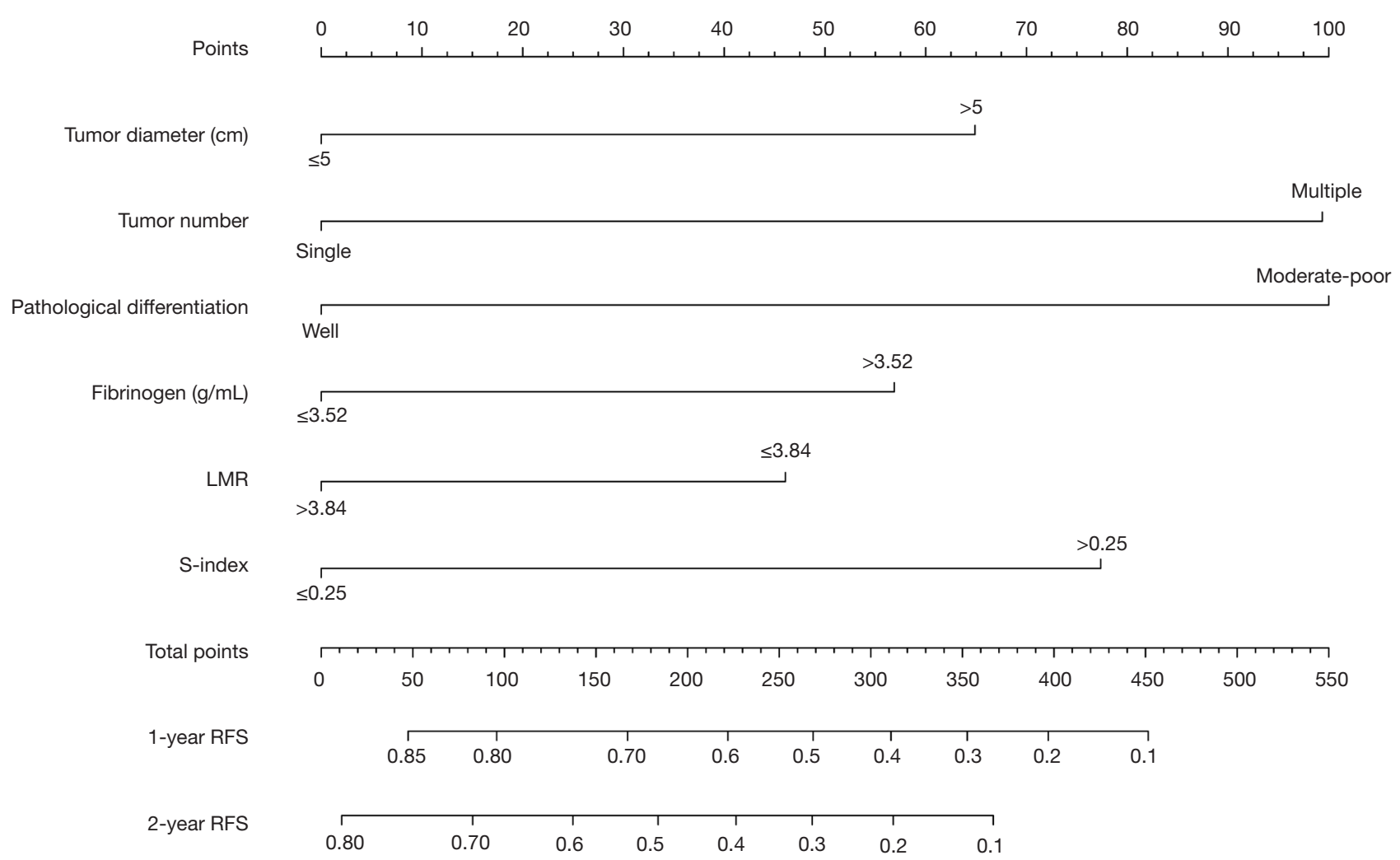

B

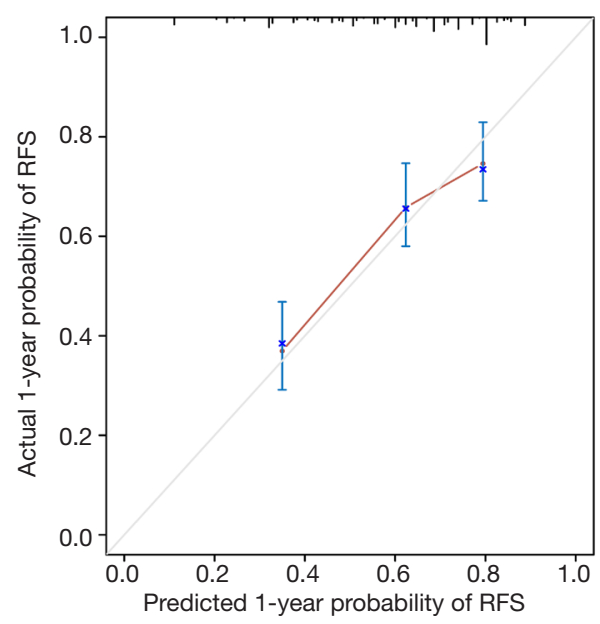

C

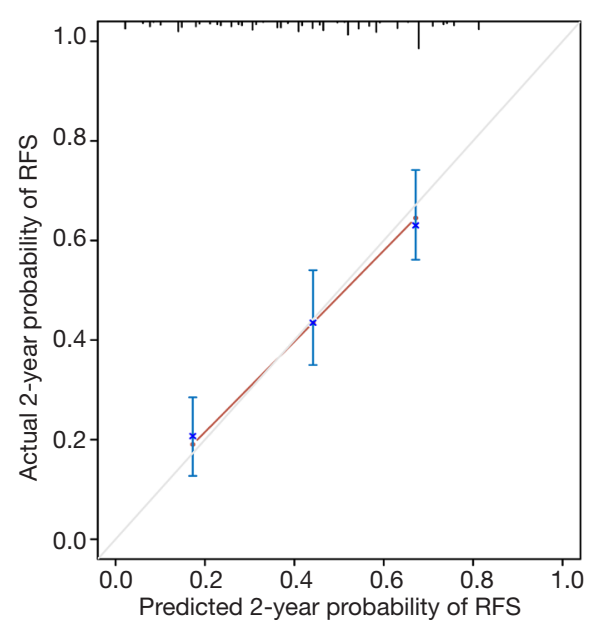

Figure 3 Nomogram for predicting the early RFS (A) and the calibration curves for predicting the 1-, and 2-year early RFS (B, and C) in HCC patients who received hepatectomy. RFS, recurrence-free survival; HCC, hepatocellular carcinoma. 
Table 4 Univariate and multivariate COX analyses for late RFS of HCC patients

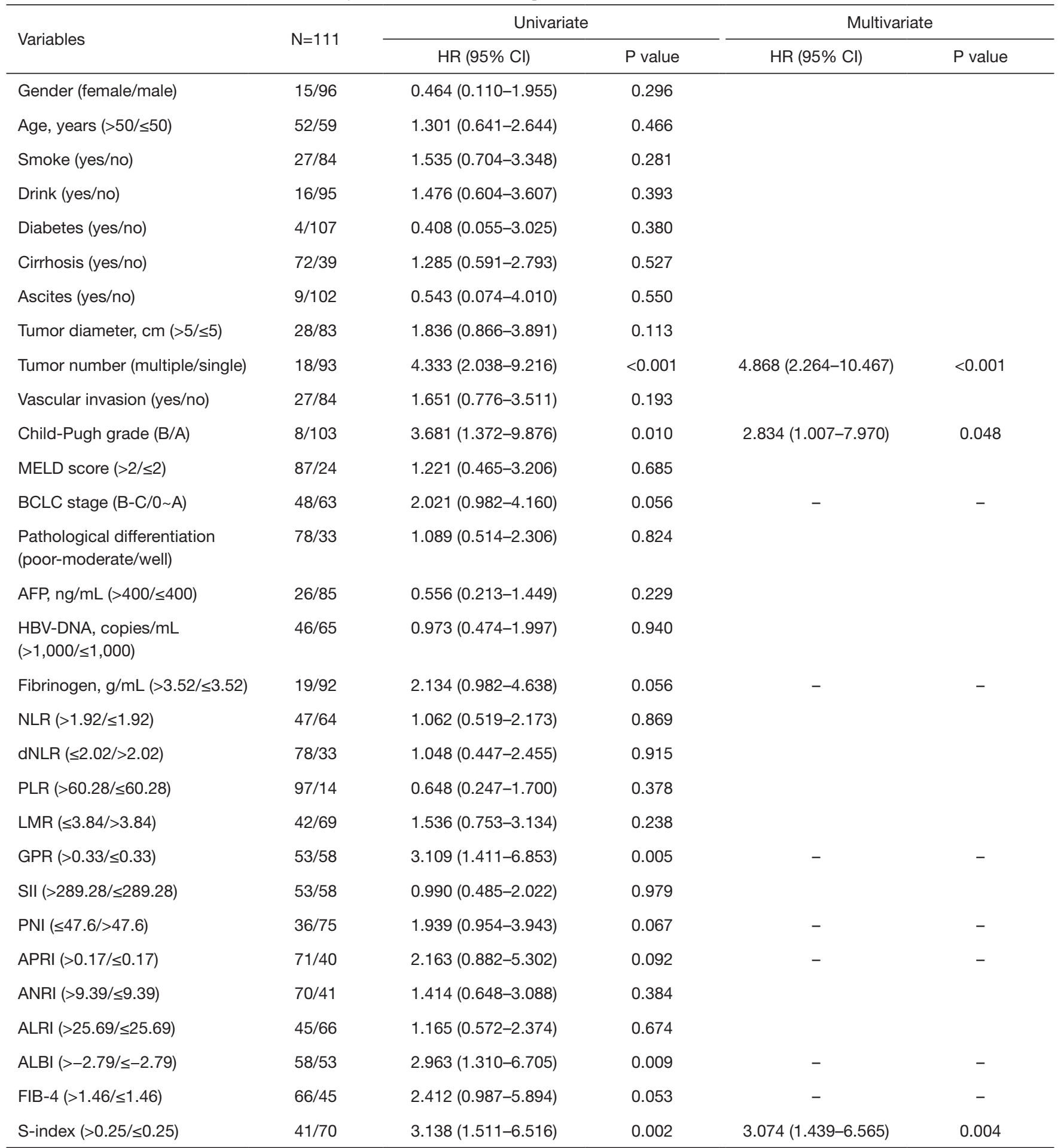

MELD, Model for End-stage Liver Disease; BCLC, Barcelona Clinic Liver Cancer; AFP, $\alpha$-fetoprotein; HBV, hepatitis B virus; NLR, neutrophil-to-lymphocyte ratio; dNLR, derived neutrophil-to-lymphocyte ratio; PLR, platelet-to-lymphocyte ratio; LMR, lymphocyteto-monocyte ratio; GPR, gamma-glutamyl transpeptidase-to-platelet ratio; SII, systemic immune-inflammation index; PNI, prognostic nutritional index; APRI, aspartate transaminase-to-platelet count ratio index; ANRI, aspartate transaminase-to-neutrophil ratio index; ALRI, aspartate transaminase-to-lymphocyte ratio index; ALBI, albumin-bilirubin; FIB-4, fibrosis index based on the four factors; RFS, recurrence-free survival. 
A

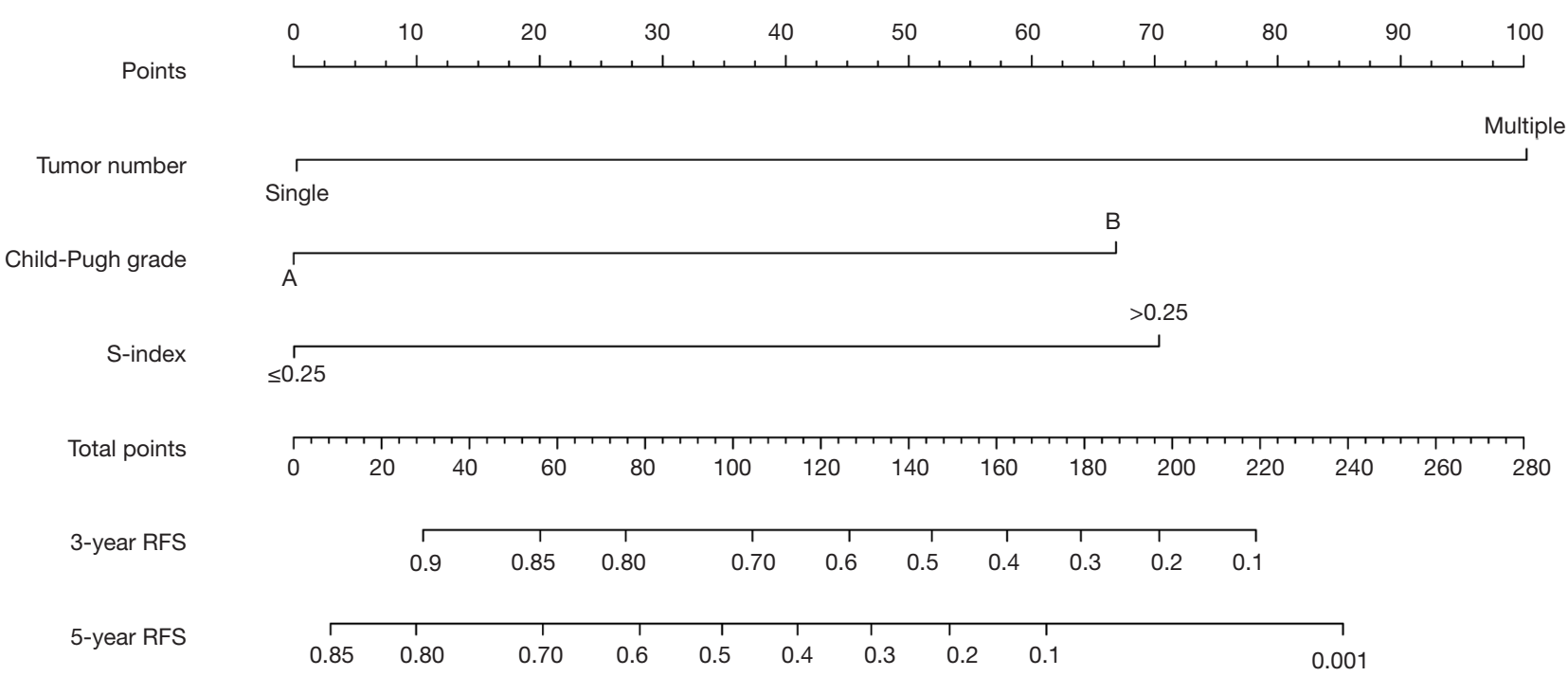

B

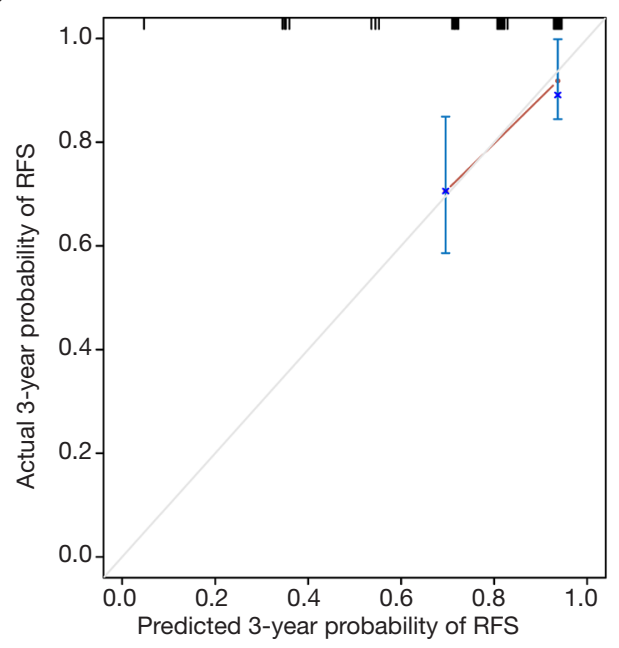

C

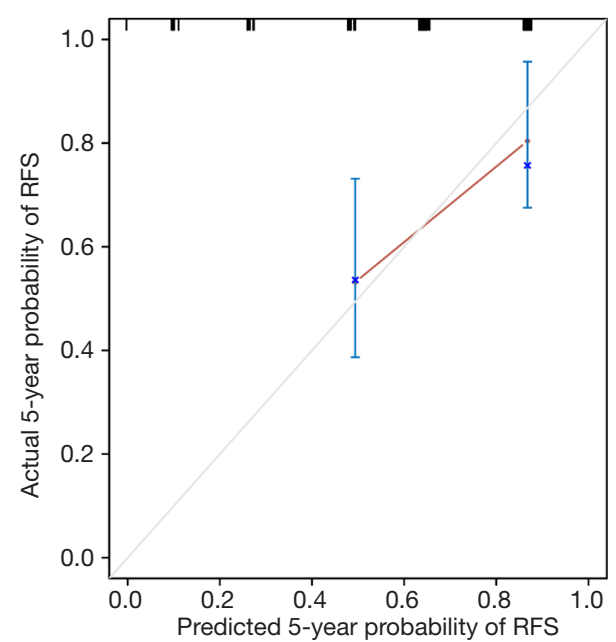

Figure 4 Nomogram for predicting the late RFS (A) and the calibration curves for predicting the 3-, and 5-year early RFS (B, and C) in HCC patients who received hepatectomy. RFS, recurrence-free survival; HCC, hepatocellular carcinoma.

Notably, there was another inflammation-related factor, the S-index, which was found to have significant prognostic value for late RFS as well as for overall and early RFS. These findings further verified the existence of distinct mechanisms for early and late recurrence of HCC. To integrate these significant independent variables derived from both clinicopathological and inflammation-related data, we constructed three nomograms as risk score models to predict early, late and overall RFS for HCC patients with $\mathrm{CHB}$ after hepatectomy. When compared with other staging systems or individual prognostic indices, the nomograms integrating inflammation-related factors showed better performance in predicting the different types of recurrence and RFS at various time points. According to the prognostic models, patients could be easily identified with different risks of recurrence as early as possible, and specific prevention measures and personal targeted therapies could then be provided promptly, which has potential benefits for improving the long-term prognosis of HCC patients.

For patients with resectable HCC, recurrence is the primary cause of poor survival rates, regardless of radical hepatectomy. Previous studies have reported that the 5-year recurrence rate of HCC almost reached $70 \%$ after surgical 
A

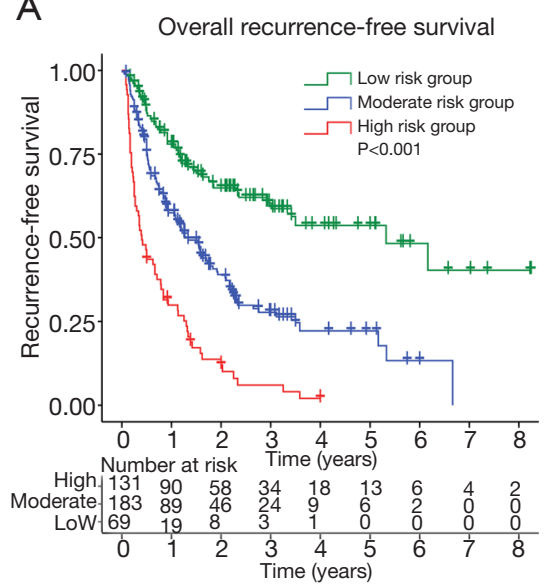

B

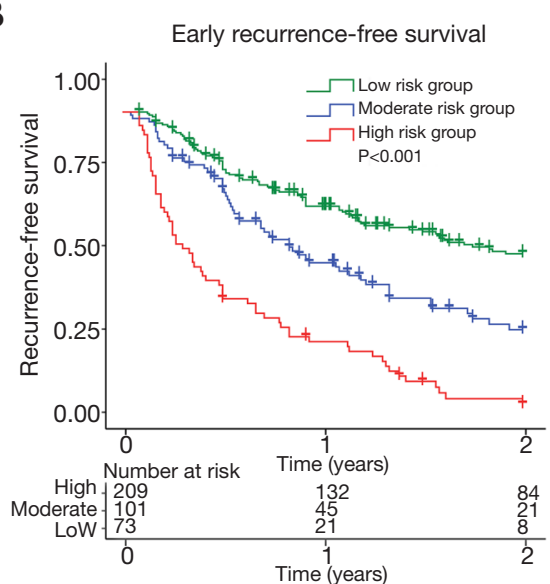

C

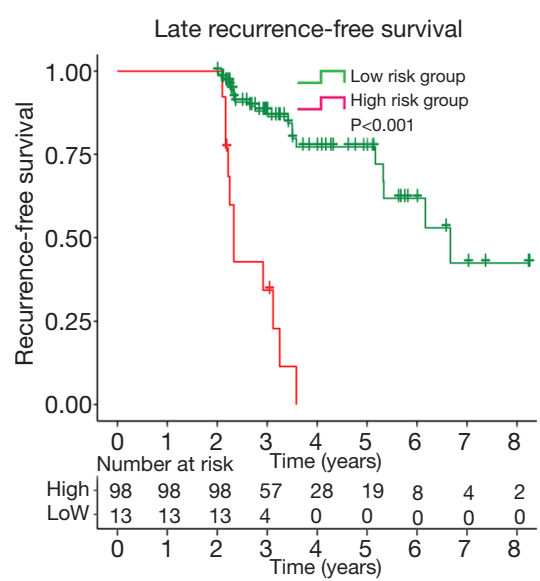

Figure 5 Kaplan-Meier curves for overall (A), early (B), and late (C) RFS in HCC patients classified into different risk groups by the established nomograms with specific NomoScores. RFS, recurrence-free survival; HCC, hepatocellular carcinoma.

A

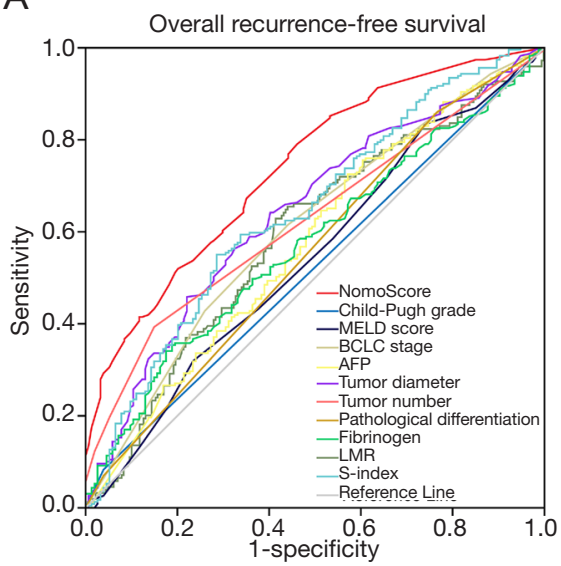

B

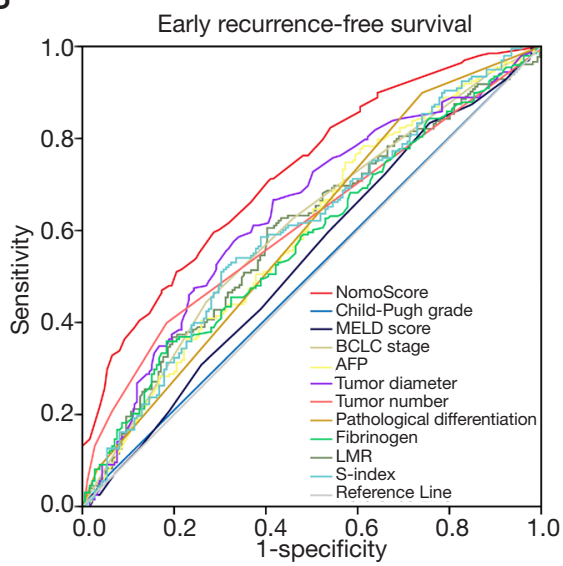

C

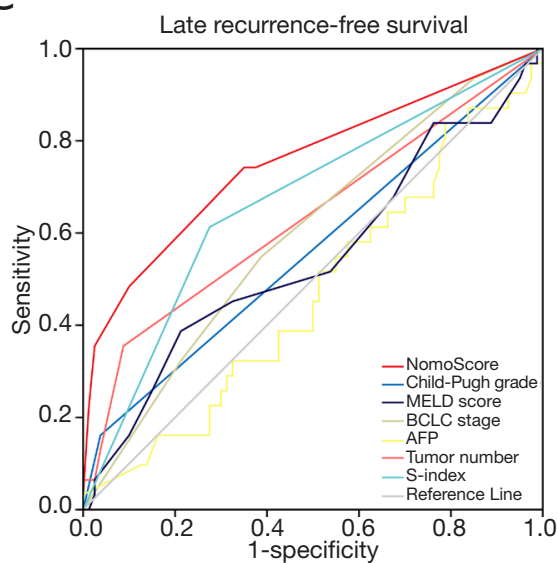

Figure 6 The ROC curves of specific nomograms and other prognostic models and independent parameters for overall (A), early (B), and late (C) RFS in HCC patients. ROC, receiver operating characteristic; RFS, recurrence-free survival; HCC, hepatocellular carcinoma.

resection (18). In the present study, a total of 383 patients who underwent liver resection for HCC were enrolled, and survival analysis showed the 5 -year overall RFS was only $26 \%$, which was in accordance with previous reports. We also evaluated the annual recurrence frequency in the cohort of recurrent patients (229 cases). The highest peak of recurrence appeared in the first year after surgery, accounting for almost $20 \%$ of all recurrence, and was also the first peak of recurrence. Thereafter, the recurrence frequency decreased until the third year, with another slightly high recurrence peak in the second postoperative year. However, the frequency increased again and contributed to a smaller peak at 4-5 years after operation.
This curve indicated a similar recrudescence pattern of early and late recurrence for HCC to that in the study conducted by Imamura et al. (5). They suggested that the early recurrence peak might be mainly due to existing metastases before surgery, whereas the majority of late recurrence was attributable to de novo lesions in the remnant liver. The cut-off time point for the different types of recurrence was determined to be post-operative 2 years.

Early recurrence accounts for the majority of total recurrence in many studies. From a total of 184 recurrent HCC patients, Imamura et al. found recurrence occurred in 123 cases $(66.8 \%)$ before 2 years (5). Portolani et al. also reported that $109(76.2 \%)$ of 143 HCC patients 
had recurrence in the first 2 years (6). In the present study, $86.5 \%$ [198] of recurrent cases belonged to earlyphase recurrence. This may be explained by the high percentage of patients with intermediate and advanced BCLC sages (54.6\%). According to the BCLC staging system, only patients with 0 -A stages are recommended to undergo surgical resection, with a candidate proportion of $5-10 \%$ (1). Emerging evidence has demonstrated the survival benefit of surgery for some HCC patients with BCLC B stage, even with advanced C stage $(19,20)$. With the extension of surgical indications, overall survival could be improved, but the recurrence rate might simultaneously increase. Though few strategies have been validated with significant effectiveness for preventing recurrence, there are several treatments for recurrent HCC, such as repeat surgery, ablation, transarterial chemoembolization (TACE), radiotherapy, targeted therapy, and recently emerging immune checkpoint inhibitors $(3,21,22)$. Hence, accurate recurrence assessments and timely treatments are imperative for patients with HCC.

Numerous studies have investigated the risk factors for recurrence in HCC patients after surgery, and many clinicopathological parameters have been identified, which could be classified into several groups, such as tumor-related characteristics (number, size, capsule, vascular invasion, pathological differentiation, stage), treatment-related factors (anatomic resection, extension of resection, surgical width, intraoperative transfusion), and host-related features (age, gender, etiology of hepatitis, cirrhosis, liver function, serological indexes) $(23,24)$. However, most studies have focused only on the overall recurrence of HCC without identifying early and late recurrence, which might neglect the influence of distinct underlying mechanisms. In the present study, however, we comprehensively investigated the respective factors contributing to overall, early, and late recurrence. We found that the risk factors for early recurrence and overall recurrence were similar, but were significantly different from those for late recurrence. Tumor number was the only tumor-related factors involved in both early and late recurrence, whereas large tumor diameter and poor-moderate pathological differentiation were identified as contributors to early recurrence. The Child-Pugh grade, a parameter reflecting the preserved liver function, was another clinicopathological indicator with predictive significance for late recurrence, but it had no relationship to early recurrence. These findings further supported the above hypothesis and may provide some insights into the derivation of early and late recurrence. Considering the complex nature of HCC, the predictive accuracy of prognosis based only on clinicopathological factors has some limitations. Identifying and utilizing new simple and effective markers for prognostic evaluation is still a pressing unmet medical need in patients with HCC.

Inflammation has been recognized as one of the major features of cancers, and its roles in tumor development, progression, and metastasis have also been well-described (8). As for HCC, an inflammation-induced malignancy, many studies have demonstrated the significant prognostic values of various inflammatory biomarkers in recurrence and survival prediction, including NLR, dNLR, PLR, LMR, and SII, which are mainly associated with systemic inflammation $(11,12,14,25)$. Other indexes such as GPR, PNI, APRI, ALRI, ANRI, FIB-4, and S-index were also developed with consideration to the active local inflammatory responses in the liver $(13,15,17,26)$. Although previous studies have tried to compare or combine these inflammation-related parameters for prognostic prediction for HCC patients, only a few studies have focused on the different types of recurrence and made specific analyses for each type. Here, we integrated the clinicopathological characteristics and above-mentioned inflammatory indicators and separately analyzed the risk factors for early and late recurrence. We found that parameters of both systemic and local inflammation contributed to early recurrence (fibrinogen, LMR and S-index), whereas only one parameter reflecting local inflammation was an independent prognostic index for late recurrence (S-index). However, Liu et al. (27) found that inflammation-related factors including NLR and APRI were independent risk factors for early recurrence, but only APRI was an inflammation-related factor for late recurrence of HCC. The difference in patient inclusion criteria (patients with and without hepatitis B) and the cut-off time point (1 year) for early and late HCC recurrence might have resulted in the diverse outcomes. Recently, Xu et al. (28) also investigated the risk factors of early and late recurrence of HCC. They adopted a 2-year cut-off time point to classify early and late recurrence of HCC, and found that ALBI was an independent risk factor for early recurrence whereas NLR correlated to late recurrence of HCC, which was different from our outcomes. In the present study, we focused mainly on the role of inflammation-related factors and enrolled many kinds of relevant parameters to the analyses, while only limited inflammation-related factors were examined in the study conducted by $\mathrm{Xu}$ et al. Hence, the results of our study provided a few novel insights 
into the characteristics of $\mathrm{HCC}$ recurrence and effective formulas for recurrence prediction.

Fibrinogen is an important factor involved in the coagulation process. Recent studies also reveal its association with the development of multiple tumors as an inflammation-related factor (29-32). Elevated plasma fibrinogen has been usually regarded as a risk factor for OS and RFS in a variety of cancers, including $\operatorname{HCC}(29,33)$. In the present study, the preoperative plasma fibrinogen showed a significant association with the overall RFS, and worse outcomes were observed in HCC patients with elevated levels $(>3.52 \mathrm{~g} / \mathrm{mL}$ ), which was consistent with previous studies. Further analysis demonstrated its prognostic significance was mainly present in early recurrence, but not in late recurrence. This may be related to the role of fibrinogen in the adhesive interaction among circulating tumor cells, platelets, and endothelial cells $(34,35)$, which could contribute to hematogenous metastasis of circulating tumor cells and lead to early recurrence. LMR is composed of the lymphocyte and monocyte counts in peripheral blood. Previous studies have demonstrated that it has a significant prognostic role in HCC, and patients with a low level of LMR presented with worse RFS and OS $(16,25,36)$, which was in accordance with our results. In the present study, we found that LMR $\leq 3.94$ was an independent risk factor for both overall recurrence and early recurrence in HCC patients who received hepatectomy. Moreover, the S-index, a simple model first constructed for predicting significant fibrosis and cirrhosis in patients with chronic hepatitis B virus (HBV) infection, was also investigated in HBV-related HCC for prognostic significance, and results showed that HCC patients with a high S-index had worse RFS and OS $(17,37)$. Our results confirmed the negative influence of the S-index in the RFS of HCC patients after surgical resection, with a high value associated with a high recurrence risk, especially late recurrence. This finding further verified the hypothesized mechanism (i.e., dependent mainly on the underlying liver status) for late recurrence.

Our study has several potential limitations. First, as a retrospective cohort study, inherent selection bias was present, and the loss to follow-up of some patients was also inevitable. Secondly, all the enrolled HCC patients in our study presented with $\mathrm{CHB}$, and several cases also had coinfection with HCV/HIV. Hence, the conclusions may have some limited application in clinical practice, and further investigation is needed for patients with other etiologies of hepatitis. Last but not least, this was a single- center study with limited sample size, and the predicted efficiency of the developed nomograms needs external validation in a multicenter study with a larger number of cases. Despite these limitations, the nomograms based on integration of inflammation-related factors still showed good discriminatory and calibration abilities for predicting postoperative recurrence in HCC patients with CHB.

In conclusion, we developed specific nomograms based on inflammation-related factors for predicting the overall, early, and late recurrence of HCC patients with CHB after hepatectomy. These easy-to-use models could be simple but effective tools for clinicians in estimating the risk of recurrence, decision-making, and planning the treatment strategies to improve the long-term prognosis of HCC patients.

\section{Acknowledgments}

Funding: This work was supported by the Guangdong Natural Science Foundation [2015A030313038, 2015A030312013]; the Science and Technology Program of Guangzhou [2014Y2-00200, 201604020001, 201508020262, 201400000001-3, 201607010024]; the Science and Technology Program of Guangdong Province [2017B020209004, 20169013]; the National 13th FiveYear Science and Technology Plan Major Projects of China [2017ZX10203205-006-001]; and the Guangdong Key Laboratory of Liver Disease Research [2017B030314027].

\section{Footnote}

Reporting Checklist: The authors have completed the STROBE reporting checklist. Available at http://dx.doi. org/10.21037/atm-20-1353

Data Sharing Statement: Available at http://dx.doi. org/10.21037/atm-20-1353

Conflicts of Interest: All authors have completed the ICMJE uniform disclosure form (available at http://dx.doi. org/10.21037/atm-20-1353). The authors have no conflicts of interest to declare.

Ethical Statement: The authors are accountable for all aspects of the work in ensuring that questions related to the accuracy or integrity of any part of the work are appropriately investigated and resolved. The study was conducted in accordance with the Declaration of Helsinki 
(as revised in 2013). The study was approved by the institutional ethics board of The Third Affiliated Hospital of Sun Yat-Sen University (NO.: 2020-02-119). Informed consent was taken from all the patients.

Open Access Statement: This is an Open Access article distributed in accordance with the Creative Commons Attribution-NonCommercial-NoDerivs 4.0 International License (CC BY-NC-ND 4.0), which permits the noncommercial replication and distribution of the article with the strict proviso that no changes or edits are made and the original work is properly cited (including links to both the formal publication through the relevant DOI and the license). See: https://creativecommons.org/licenses/by-nc-nd/4.0/.

\section{References}

1. Forner A, Reig M, Bruix J. Hepatocellular carcinoma. Lancet 2018;391:1301-14.

2. Bray F, Ferlay J, Soerjomataram I, et al. Global cancer statistics 2018: GLOBOCAN estimates of incidence and mortality worldwide for 36 cancers in 185 countries. CA Cancer J Clin 2018;68:394-424.

3. Yang JD, Hainaut P, Gores GJ, et al. A global view of hepatocellular carcinoma: trends, risk, prevention and management. Nat Rev Gastroenterol Hepatol 2019;16:589-604.

4. Llovet JM, Zucman-Rossi J, Pikarsky E, et al. Hepatocellular carcinoma. Nat Rev Dis Primers 2016;2:16018.

5. Imamura $\mathrm{H}$, Matsuyama $\mathrm{Y}$, Tanaka $\mathrm{E}$, et al. Risk factors contributing to early and late phase intrahepatic recurrence of hepatocellular carcinoma after hepatectomy. J Hepatol 2003;38:200-7.

6. Portolani N, Coniglio A, Ghidoni S, et al. Early and late recurrence after liver resection for hepatocellular carcinoma: prognostic and therapeutic implications. Ann Surg 2006;243:229-35.

7. Wu JC, Huang YH, Chau GY, et al. Risk factors for early and late recurrence in hepatitis B-related hepatocellular carcinoma. J Hepatol 2009;51:890-7.

8. Grivennikov SI, Greten FR, Karin M. Immunity, inflammation, and cancer. Cell 2010;140:883-99.

9. Chechlinska M, Kowalewska M, Nowak R. Systemic inflammation as a confounding factor in cancer biomarker discovery and validation. Nat Rev Cancer 2010;10:2-3.

10. Liao R, Peng C, Li M, et al. Comparison and validation of the prognostic value of preoperative systemic immune cells in hepatocellular carcinoma after curative hepatectomy. Cancer Med 2018;7:1170-82.

11. Zheng J, Cai J, Li H, et al. Neutrophil to Lymphocyte Ratio and Platelet to Lymphocyte Ratio as Prognostic Predictors for Hepatocellular Carcinoma Patients with Various Treatments: a Meta-Analysis and Systematic Review. Cell Physiol Biochem 2017;44:967-81.

12. Yang R, Chang Q, Meng X, et al. Prognostic value of Systemic immune-inflammation index in cancer: A metaanalysis. J Cancer 2018;9:3295-302.

13. Wang Y, Sun K, Shen J, et al. Novel Prognostic Nomograms Based on Inflammation-Related Markers for Patients with Hepatocellular Carcinoma Underwent Hepatectomy. Cancer Res Treat 2019;51:1464-78.

14. Zhou D, Zhang Y, Xu L, et al. A monocyte/granulocyte to lymphocyte ratio predicts survival in patients with hepatocellular carcinoma. Sci Rep 2015;5:15263.

15. Zhang Y, Wang R, Yang X. FIB-4 index serves as a noninvasive prognostic biomarker in patients with hepatocellular carcinoma: A meta-analysis. Medicine (Baltimore) 2018;97:e13696.

16. Song W, Tian C, Wang K, et al. The pretreatment lymphocyte to monocyte ratio predicts clinical outcome for patients with hepatocellular carcinoma: A metaanalysis. Sci Rep 2017;7:46601.

17. Gu J, Zhang X, Wang Z, et al. Simplified nomograms based on platelet-associated models for survival prediction in Asian hepatocellular carcinoma patients after surgery. Surg Oncol 2019;30:131-8.

18. Tabrizian P, Jibara G, Shrager B, et al. Recurrence of hepatocellular cancer after resection: patterns, treatments, and prognosis. Ann Surg 2015;261:947-55.

19. Galle PR, Tovoli F, Foerster F, et al. The treatment of intermediate stage tumours beyond TACE: From surgery to systemic therapy. J Hepatol 2017;67:173-83.

20. Hyun MH, Lee YS, Kim JH, et al. Hepatic resection compared to chemoembolization in intermediate- to advanced-stage hepatocellular carcinoma: A meta-analysis of high-quality studies. Hepatology 2018;68:977-93.

21. Yoh T, Seo S, Taura K, et al. Surgery for Recurrent Hepatocellular Carcinoma: Achieving Long-term Survival. Ann Surg 2019:1. [Epub ahead of print]. doi: 10.1097/ SLA.0000000000003358.

22. Bruix J, Takayama T, Mazzaferro V, et al. Adjuvant sorafenib for hepatocellular carcinoma after resection or ablation (STORM): a phase 3, randomised, double-blind, placebo-controlled trial. Lancet Oncol 2015;16:1344-54.

23. Toyoda H, Kumada T, Tada T, et al. Prognostic 
significance of a combination of pre- and post-treatment tumor markers for hepatocellular carcinoma curatively treated with hepatectomy. J Hepatol 2012;57:1251-7.

24. Sasaki Y, Yamada T, Tanaka H, et al. Risk of recurrence in a long-term follow-up after surgery in 417 patients with hepatitis B- or hepatitis C-related hepatocellular carcinoma. Ann Surg 2006;244:771-80.

25. Lin ZX, Ruan DY, Li Y, et al. Lymphocyte-to-monocyte ratio predicts survival of patients with hepatocellular carcinoma after curative resection. World J Gastroenterol 2015;21:10898-906.

26. Qin W, Wang L, Hu B, et al. A Novel Score Predicts HBV-Related Hepatocellular Carcinoma Recurrence After Hepatectomy: a Retrospective Multicenter Study. J Gastrointest Surg 2019;23:922-32.

27. Liu Y, Wang ZX, Cao Y, et al. Preoperative inflammationbased markers predict early and late recurrence of hepatocellular carcinoma after curative hepatectomy. Hepatobiliary Pancreat Dis Int 2016;15:266-74.

28. Xu W, Li R, Liu F. Novel Prognostic Nomograms for Predicting Early and Late Recurrence of Hepatocellular Carcinoma After Curative Hepatectomy. Cancer Manag Res 2020;12:1693-712.

29. Dai T, Peng L, Lin G, et al. Preoperative elevated plasma fibrinogen level predicts tumor recurrence and poor prognosis in patients with hepatocellular carcinoma. J Gastrointest Oncol 2019;10:1049-63.

30. Yu X, Hu F, Yao Q, et al. Serum fibrinogen levels are positively correlated with advanced tumor stage and

Cite this article as: Dai T, Deng M, Ye L, Lin G, Liu R, Deng Y, Li R, Liu W, Li H, Yang Y, Chen G, Wang G. Nomograms based on clinicopathological factors and inflammatory indicators for prediction of early and late recurrence of hepatocellular carcinoma after surgical resection for patients with chronic hepatitis B. Ann Transl Med 2021;9(1):12. doi: 10.21037/atm-20-1353 poor survival in patients with gastric cancer undergoing gastrectomy: a large cohort retrospective study. BMC Cancer 2016;16:480.

31. Li H, Zhao T, Ji X, et al. Hyperfibrinogenemia predicts poor prognosis in patients with advanced biliary tract cancer. Tumour Biol 2016;37:3535-42.

32. Perisanidis C, Psyrri A, Cohen EE, et al. Prognostic role of pretreatment plasma fibrinogen in patients with solid tumors: A systematic review and meta-analysis. Cancer Treatment Reviews 2015;41:960-70.

33. Huang G, Jiang H, Lin Y, et al. Prognostic value of plasma fibrinogen in hepatocellular carcinoma: a meta-analysis. Cancer Manag Res 2018;10:5027-41.

34. Palumbo JS, Kombrinck KW, Drew AF, et al. Fibrinogen is an important determinant of the metastatic potential of circulating tumor cells. Blood 2000;96:3302-9.

35. Languino LR, Plescia J, Duperray A, et al. Fibrinogen mediates leukocyte adhesion to vascular endothelium through an ICAM-1-dependent pathway. Cell 1993;73:1423-34.

36. Li GJ, Ji JJ, Yang F, et al. Preoperative lymphocyte-tomonocyte ratio predicts survival in primary hepatitis B virus-positive hepatocellular carcinoma after curative resection. Onco Targets Ther 2017;10:1181-9.

37. Zhou K, Gao CF, Zhao YP, et al. Simpler score of routine laboratory tests predicts liver fibrosis in patients with chronic hepatitis B. J Gastroenterol Hepatol 2010;25:1569-77. 
Table S1 The formulas of inflammation-related indexes

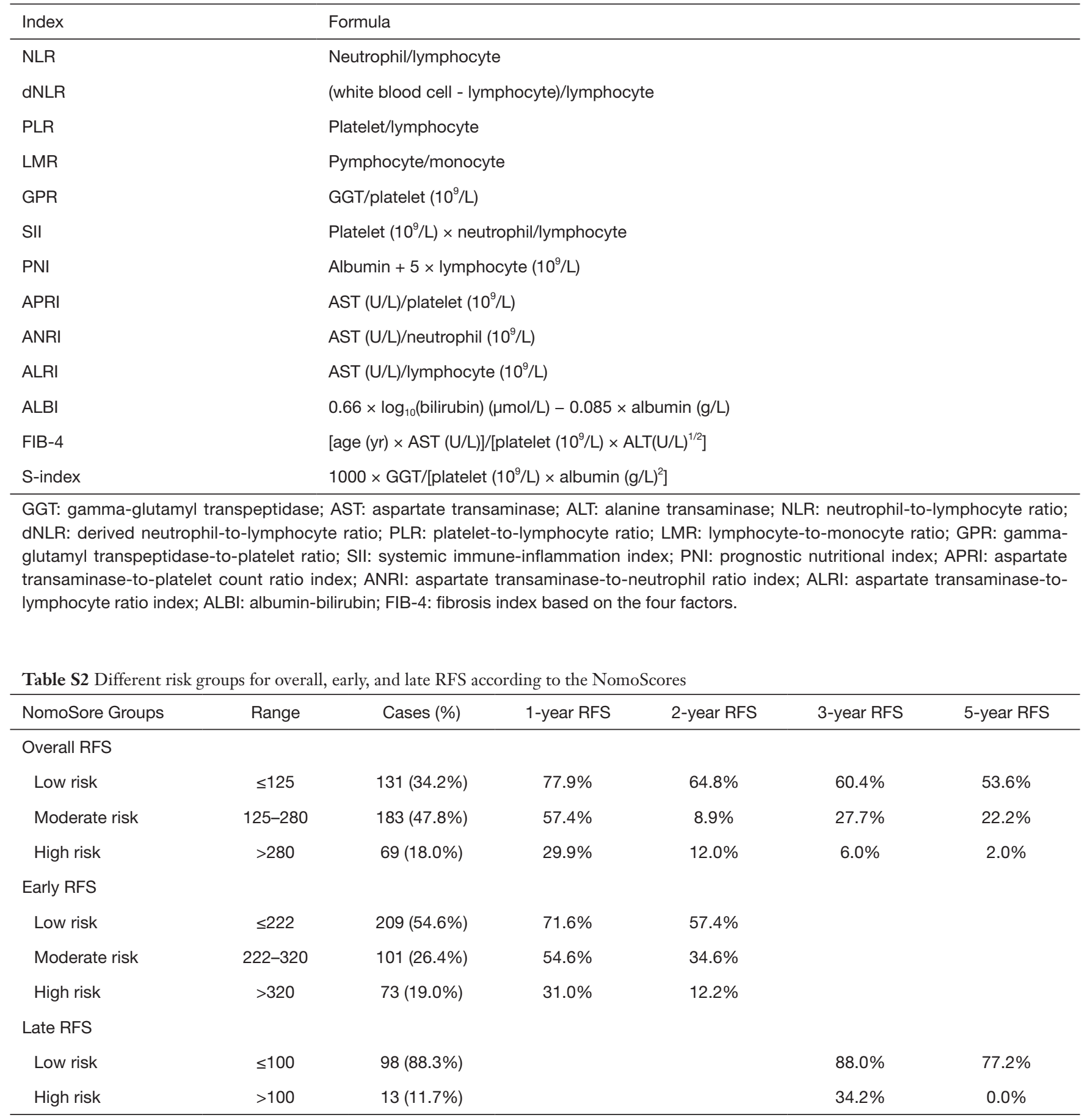

RFS: recurrence-free survival. 
A

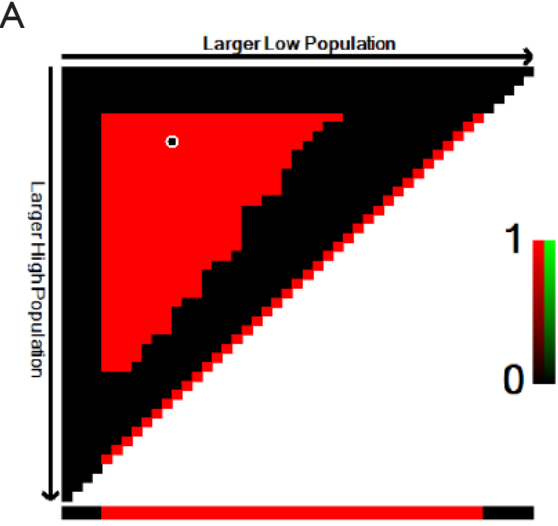

D

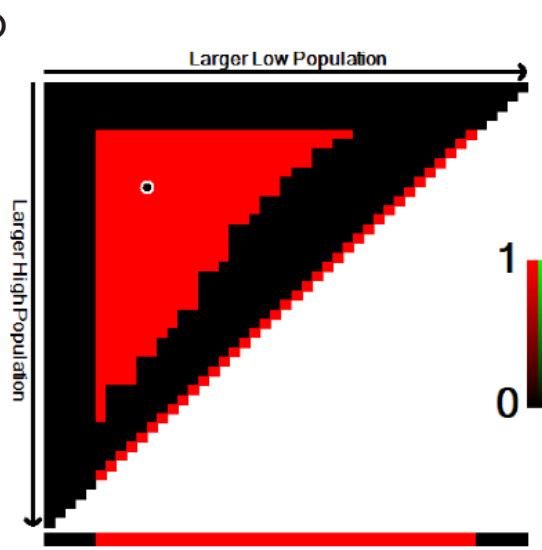

G

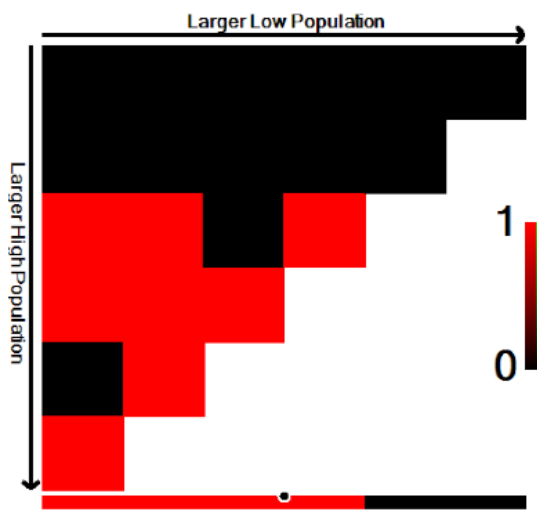

B

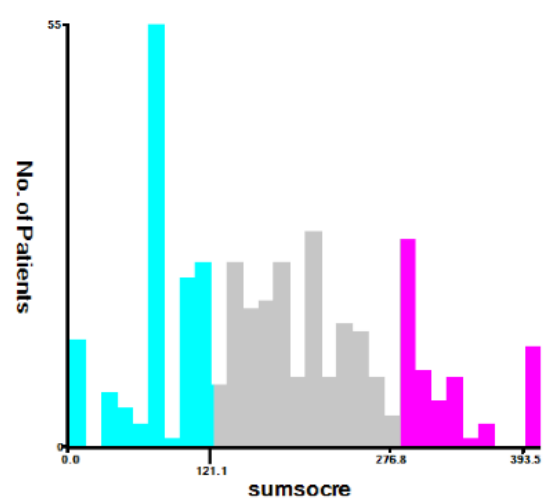

E

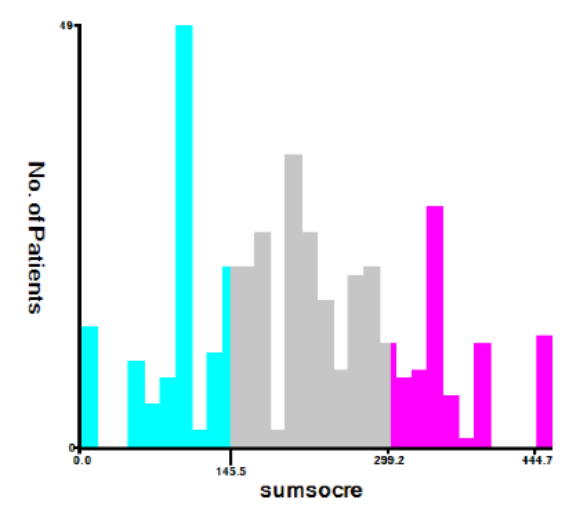

$\mathrm{H}$

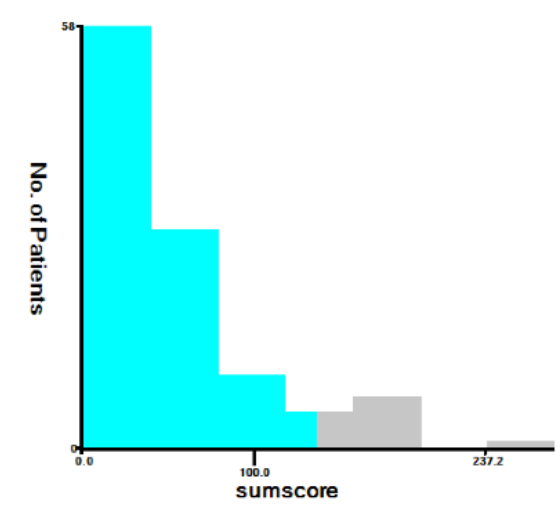

C

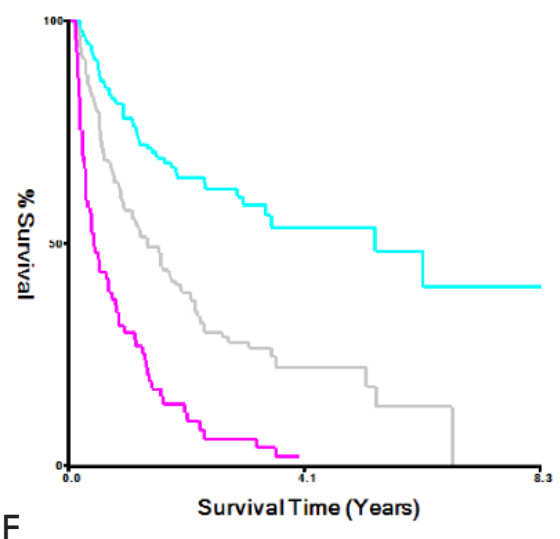

$\mathrm{F}$

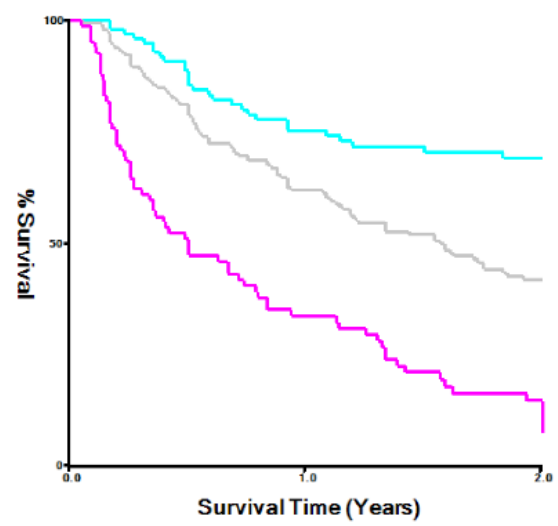

I

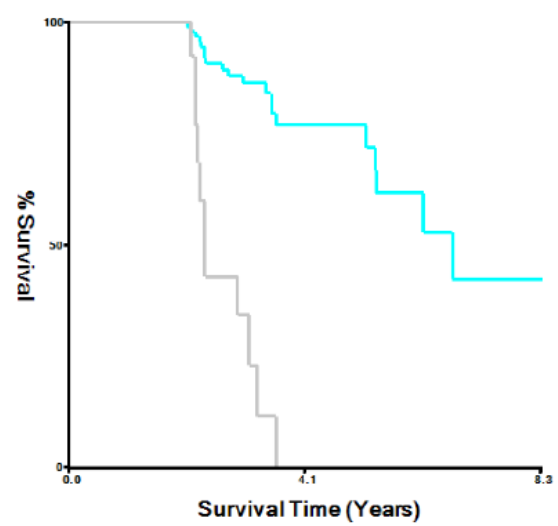

Figure S1 The cut-off point identification of the NomoScores for overall (A, B, and C), early (D, E, and F), and late (G, H, and I) RFS produced by X-tile plots. RFS: recurrence-free survival. 